\title{
Image Encryption Based on Phase Decomposition Technique
}

\author{
Katsuhiro Uno ${ }^{\mathrm{a},{ }^{*}}$, Hoang Hoa Tien Dungr ${ }^{\mathrm{a}}$ \\ ${ }^{a}$ Ibaraki University, 4-12-1 Nakanarusawa, Hitachi 316-8511, Japan \\ *Corresponding Author: katsuhiro.uno.catsin3@ vc.ibaraki.ac.jp
}

\begin{abstract}
Novel techniques for image encryption, which is based on a phase decomposition of the complex amplitude, and for decryption with an interferometry were proposed. The phase decomposition was successfully performed by retarding or progressing the random phase along the pixel brightness information of the image that should be encrypted. The obtained images that were encrypted were purely random phase images with constant amplitude, so that no one could see the meaning of the image. The hidden image could be recovered only by the interferometric technique. The decrypted image was exactly the same image as the original one without any noise. Our technique can be applied not only to binary images but also to grayscale images. We confirmed our technique in the computer simulation.
\end{abstract}

Keywords: visual cryptography, phase decomposition, interferometric technique, image encryption, decryption.

\section{Introduction}

Visual cryptography (VC) was invented by Naor and Shamir ${ }^{(1)}$ in 1995. They decomposed an image into $n$ transparencies that look like random patterns. Each transparency is called as "share". The image is recognizable only if $k$ 's out of $n$ shares are stacked together. Each share expresses one pixel of the image by some subpixels with black or white. Black and white subpixels are coded so that the stack result produced black if the image pixel is black. The stack result of subpixels looks grey when the image pixel is white. If we can distinguish the grey from black, we are able to recognize the image. Subpixel decomposition accompanies a pixel expansion, which leads to share size bigger than the original image.

The random-grid (RG) cryptography invented by $\mathrm{O}$. Kafri and E. Keren ${ }^{(2)}$ is one of the encryption methods without pixel expansion. They used the random grid that consists of randomly distributed black and white pixels as one of the share. The other share was constructed by assigning the pixel brightness same as the random grid provided that the image had a white pixel, and setting the complementary brightness provided that the image pixel was black. Stacking transparencies of shares produces black portions where complementary pixels are assigned and grey portions where identical pixels are assigned. We can recognize the decrypted image as contrast variance between black and grey. The contrast of the decrypted image was very low, because the white area of the encrypting image contains randomly distributed black pixels as a noise.

Many researchers have devoted to the contrast enhancement and the reduction of pixel expansion for $\mathrm{VC}^{(3)-(9)}$. However, the image contrast is not enough to apply to the industrial level. Moreover, encryptable image has been limited to the binary type of the image.

We propose a very new technique for VC that uses coherent optics. We invented the idea that decomposes pixel brightness of the image into randomly distributed phasors of wave fields for encryption. Shares made by our proposed method have uniform transmittance with random phase, so that no one could find any image on the display, even random noise. The encrypted image can be reproduced by the interferometric technique. As far as we know, S-S. Lee et al. ${ }^{(10)}$ were first ones that used the interferometric technique to VC. They have still used binary images and binary phase coding. Our technique uses continuous phase coding and is applicable to any type of images, such as grayscale and color images. Apart from some diffraction effects, the decrypted image by our technique will have no noise and be exactly the same as original image.

\section{Theory}




\subsection{Image encryption method}

All images that are displayed on the TV monitor or the projector are represented by some pixel brightness whether monochromatic images or not. Even in color images, pixel brightness values are assigned for each primary color component, i.e. red, green, and blue. The pixel brightness is converted to the light intensity when viewing the images on the monitor or projection.

Let us consider that the light intensity transmitted through or reflected from the display device is represented by $I(i, j)$. Here, indices $i$ and $j$ indicate $i$ th row and $j$ th column of the image pixel. The light intensity represents a light energy incident into unit area per second and is proportional to square modulus of the electric or magnetic vector. In the isotropic media, one allows to deal with only one component of the electric or magnetic vector as a scalar wave field, without loss of generality. We denote the scalar wave field as $U(x, y)$. The field has two variables, i.e. amplitude $A$ and phase $\phi$, and can be expressed as $U=A \exp (j \phi)$. Then, the intensity is related to the amplitude as $I=|U|^{2}=A^{2}$ and not related to its phase. Therefore, one degree of freedom will be added to express the light intensity, when expressing it as the wave field.

We consider the phase $\phi$ to be a random variable of which value uniformly ranges from $-\pi$ to $\pi$. Then, the amplitude $A$ is equal to the square root of the light intensity of the image, i.e. $A=\sqrt{I}$. If the wave field transmitted or reflected from the display device has the same profile as $U$, the light intensity received by human eye or projection screen will depict the original image regardless of the feature of the phase distribution because of $|U|^{2}=|A \exp (j \phi)|^{2}=A^{2}=I$.

It is known that the liquid crystal display device can alter the phase of the wave field along with the pixel gradation of the image. If the amplitude transmittance or reflectance of the device is $r(0 \leq r \leq 1)$, and the maximum light intensity of the incident wave is $I_{\max }$, then the transmitted or reflected wave amplitude becomes $A_{t}=r \sqrt{I_{\max }}$.

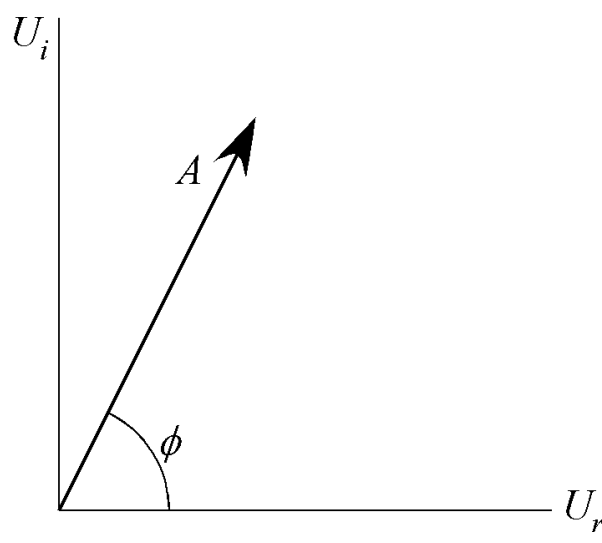

Fig. 1 A phasor denoting the wave field.

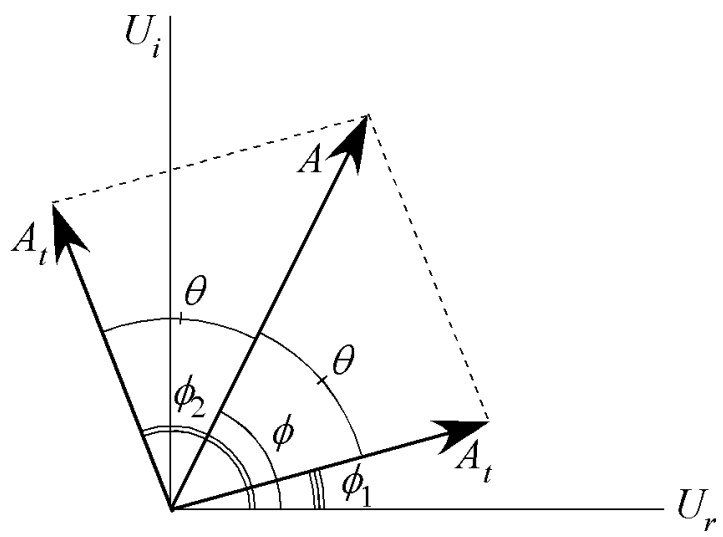

Fig. 2 A phasor description for two wave decomposition.

Now we denote the wave field $U$ as a phasor of which length and phase angle are $A$ and $\phi$, respectively, as shown in Fig. 1. Let us consider how to compose the same phasor using two different phasors of which lengths are $A_{t}$. This can be easily implemented as shown in Fig. 2. The first phasor $U_{1}$ has a length of $A_{t}$ and a phase angle of $\phi_{1}$, i.e. $U_{1}=A_{t} \exp \left(j \phi_{1}\right)$. Similarly, the second phasor $U_{2}$ has a length of $A_{t}$ and a phase angle of $\phi_{2}$, i.e. $U_{2}=A_{t} \exp \left(j \phi_{2}\right)$. When denoting the angle between the phasor $U$ and the first phasor $U_{1}$ as $\theta$, the same angle becomes between $U$ and the second phasor $U_{2}$. From the geometry of Fig. 2, the angle $\phi_{1}$ and $\phi_{2}$ are related to the random phase $\phi$ and $\theta$ as

$$
\phi_{1}=\phi-\theta \text {, }
$$




$$
\phi_{2}=\phi+\theta \text {. }
$$

Moreover, the following cosine theorem can be applied as

$$
A^{2}+A_{t}^{2}-2 A A_{t} \cos \theta=A_{t}^{2} \text {. }
$$

Then, we obtain

$$
\theta=\cos ^{-1}\left(\frac{A}{2 A_{t}}\right) .
$$

It is noted that the phase angles $\phi_{1}$ and $\phi_{2}$ are also random variables since $\phi$ is random. Therefore, two wave fields having phasors $U_{1}$ and $U_{2}$ have random phase profiles. However, their amplitudes are the constant value of $\left|U_{1}\right|=\left|U_{2}\right|=A_{t}$, so that no one is not able to see what the device displays since we can only see the constant intensity of $\left|U_{1}\right|^{2}=\left|U_{2}\right|^{2}=A_{t}^{2}=r^{2} I_{\max }$.

\section{$2.2 \quad$ Image decryption}

Image decryption will be performed only by the interferometric technique. This means that two wave fields are superposed at the observation plane. When the wave field $U_{1}$ is added to $U_{2}$ coaxially, the total wave field $V$ is written as

$$
\begin{aligned}
V & =U_{1}+U_{2} \\
& =A_{t}\left[\exp \left(j \phi_{1}\right)+\exp \left(j \phi_{2}\right)\right]^{\cdot}
\end{aligned}
$$

Since only the light intensity is detectable for all existing detectors or human eyes, we will receive the signal of square modulus of the wave field as $|V|^{2}$. Substituting eqs. (1), (2), and (4) into eq. (5) and square modulus, we finally obtain the original image intensity profile as

$$
\begin{aligned}
|V|^{2} & =A_{t}^{2} \mid \exp [j(\phi-\theta)]+\exp [j(\phi+\theta)]^{2} \\
& =2 A_{t}^{2}[1+\cos (2 \theta)] \\
& =4 A_{t}^{2} \cos ^{2} \theta \\
& =4 A_{t}^{2}\left(\frac{A}{2 A_{t}}\right)^{2}=A^{2}=I
\end{aligned}
$$

It should be noted that we do not set any premise about the intensity profile $I(x, y)$, so that all kind of images not only binary images but also gray scale images can be encryptable.

\section{Simulation Results}

Based on the theory of previous section, we demonstrated the image encryption and decryption. Two kinds of images were used for encryption. One is a binary image as shown in Fig. 3 and the other is a gray scale image as shown in Fig.4.

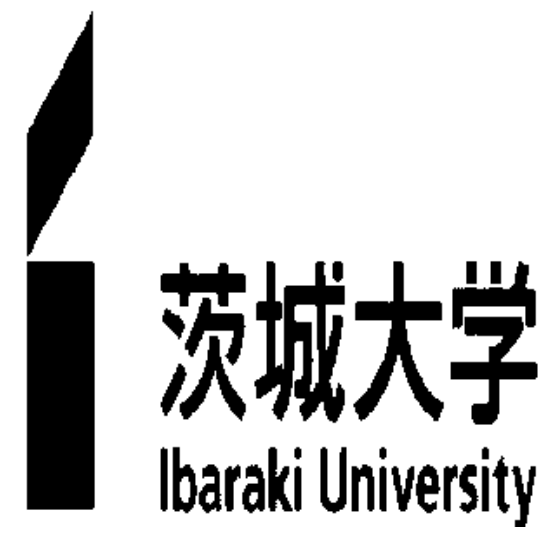

Fig. 3 A binary image for encryption.

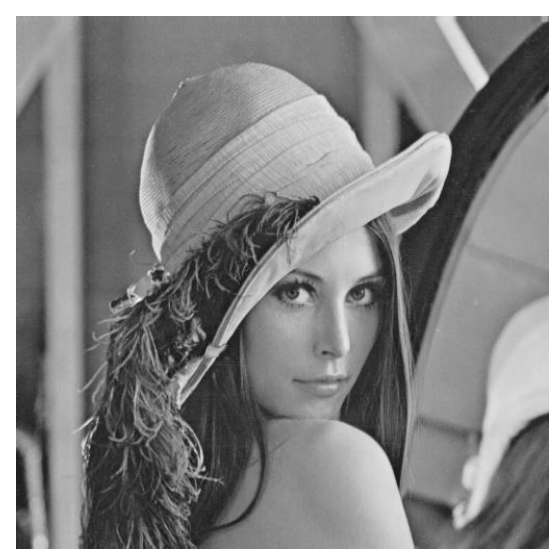

Fig. 4 A grayscale image for encryption.

Procedures for encryption and decryption are shown as flow charts in Fig. 5. For comparison, the encryption and decryption process of the random grid method are also shown in Fig. 6. In the encryption, we set parameters, $I_{\max }=1$ and $r=1$, so that $A_{t}=1$. As shown in Fig. 5 and Fig. 6, the number of calculation steps of the proposed method is larger than that of the random grid. However, a bitwise exclusive OR calculation in the random grid method is heavier than other operation. When we used Scilab program for calculation, computation time of the random grid method was about 10 times larger than the proposed method. Moreover, the decryption by the proposed method can be performed optically so that the decrypted image can be obtained much faster than the random grid method. 


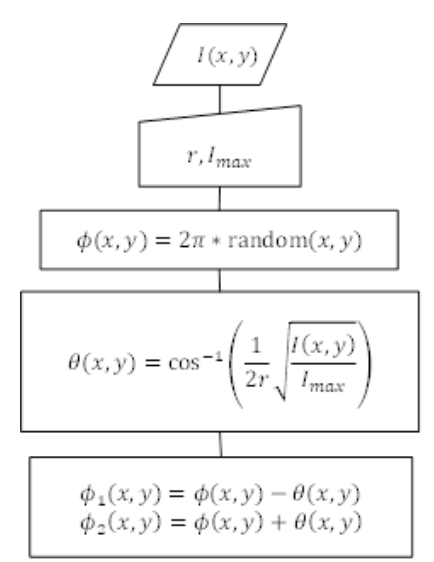

(a)

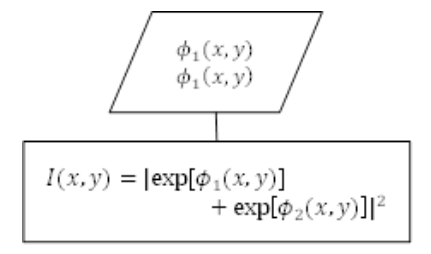

(b)
Fig. 5 The flow chart of the proposed method for (a) encryption and (b) decryption.

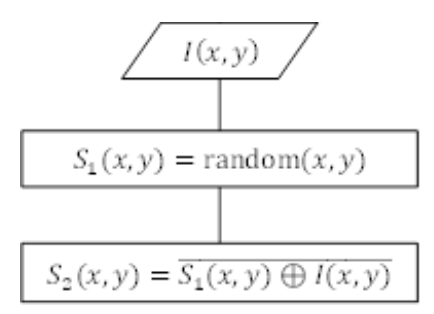

(a)

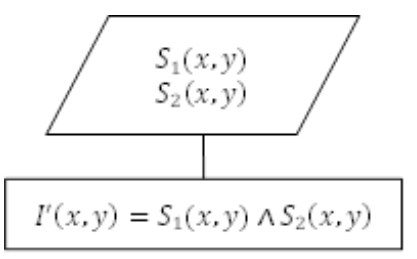

(b)
Fig. 6 The flow chart of the random grid method for (a) encryption and (b) decryption.

According to the encrypted method shown in Fig. 5(a), we decomposed images shown in Fig. 3 and Fig. 4 into two randomly distributed phase profiles, respectively. The phase profiles made from the binary image in Fig. 3 are shown in Fig. 7. Phase components of the wave fields were randomized completely. Needless to say, the amplitude components of the wave fields are constant value of $A_{t}=1$ (not shown in the figure). Therefore, no one can figure out the image contents.

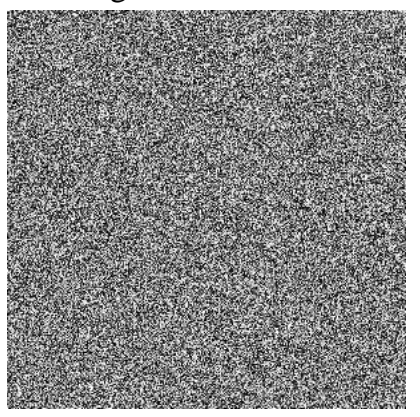

(a)

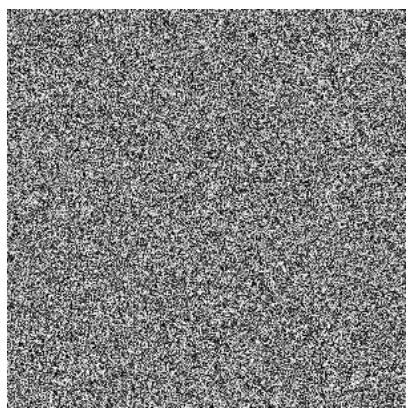

(b)
Fig. 7 Phase profiles, (a) $\phi_{1}$ and (b) $\phi_{2}$ of two wave fields, $U_{1}$ and $U_{2}$ decomposed from the binary image in Fig. 3.
We calculated the histogram of the original image and one of the phase profiles $\phi_{1}$ as shown in Fig. 8. The histogram of phase profile is fairly uniform and is significantly different from that of the original image.

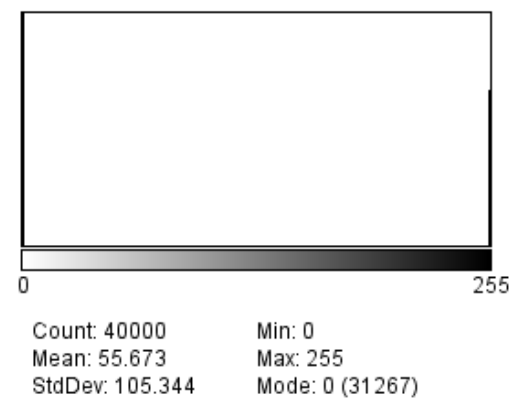

(a)
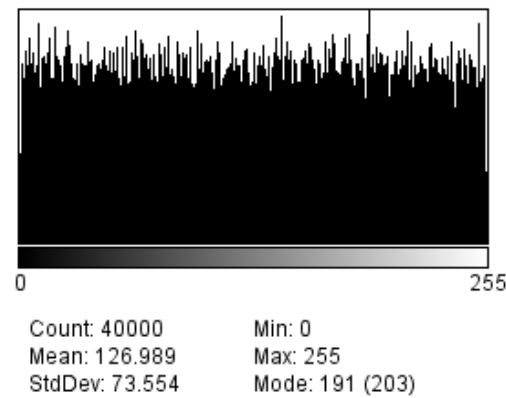

(b)

Fig. 8 The histogram of (a) the original binary image and (b) decomposed phase profile.

When the two wave fields, $U_{1}$ and $U_{2}$, were interferometrically superposed to pixel by pixel according to eq. (5), i.e.

$$
\begin{aligned}
V(i, j) & =U_{1}(i, j)+U_{2}(i, j) \\
& =A_{t} \exp \left[j \phi_{1}(i, j)\right]+A_{t} \exp \left[j \phi_{2}(i, j)\right],
\end{aligned}
$$

the intensity profile $I=|V|^{2}$ became completely the same as original image. The optical decryption can be performed by the interferometric apparatus shown in Fig. 9. A laser source is divided into two beams impinging on a LCD panel. LCD depicts two phase profiles (share1 and share2) as shown in Fig. 7 where two arms of the laser beams go through. Two beams are coherently superposed at the mirror 3 and finally create the decrypted image at the screen. Unfortunately, owing to the current resource limitation in our laboratory, we just made some numerical simulations to verify the feasibility and effectiveness of the proposed method. 


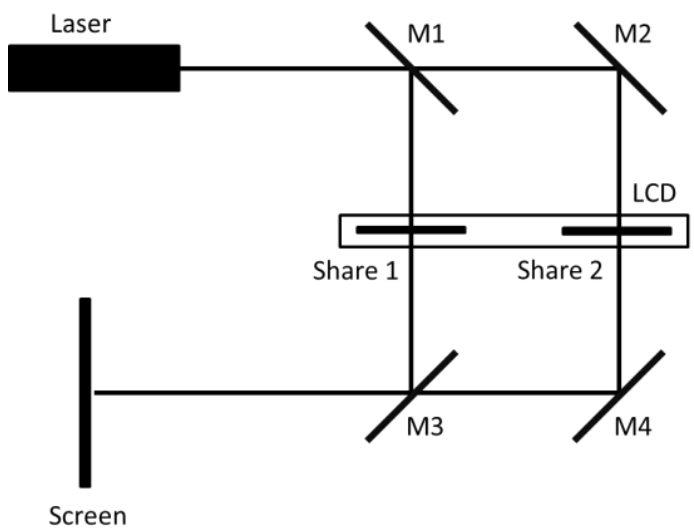

Fig. 9 Optically decrypting setup.

The interferogram calculated by eq. (7) is shown in Fig. 10. By the interferometric superposition, the decoding error, which is inherent in the conventional visual secret sharing, was completely eliminated from the decrypted image.

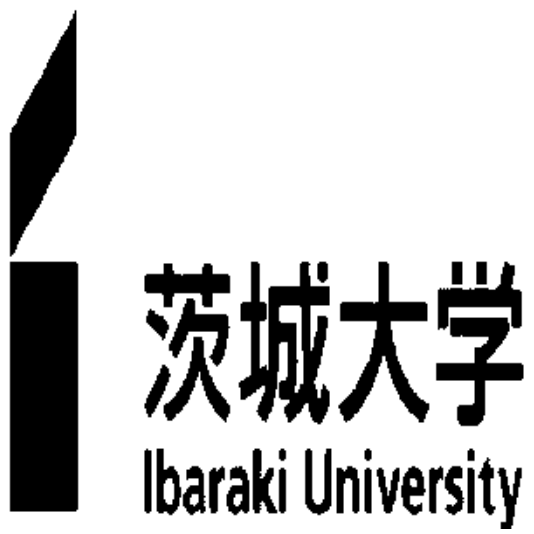

Fig. 10 Decrypted image by interferometric superposition between Fig. 7(a) and (b).

Another example is shown in Fig. 11 as a result of phase profiles of two wave fields encrypted from the grayscale image of Fig. 4. The interferogram decrypted from two wave fields with these phase profile is shown in Fig. 12. The decrypted image was completely the same as the original one. The histograms of encrypted phase profile $\phi_{1}$ and the original image are shown in Fig. 13. The histogram of the phase profile is again uniform and is greatly different from the original's one.

We also estimated sensitivity against a noise. We added the Gaussian noise with zero mean and 0.1 standard deviation to one of the share. Conventional random grid method was used for comparison. Decoded images from two shares with noise are shown in Fig. 14. As shown in those figures, the decrypted image by the proposed method is clearer than that of the random grid method. The mean square error (MSE) of two images is measured for estimating noise sensitivity, which is defined as

$$
\operatorname{MSE}\left(I_{1}, I_{2}\right)=\frac{1}{M N} \sum_{i=1}^{M} \sum_{j=1}^{N}\left|I_{2}(i, j)-I_{1}(i, j)\right|^{2} .
$$

The MSE between the original image and decrypted image is tabulated in Table 1. The error of proposed method is significantly low, when the standard deviation $\sigma$ of Gaussian noise is 0 , i.e. no noise. However, the proposed method is more sensitive to the noise, since the MSE at $\sigma=0.1$ is $10^{28}$ times larger than that of no noise while MSEs of the random grid are almost unaltered.

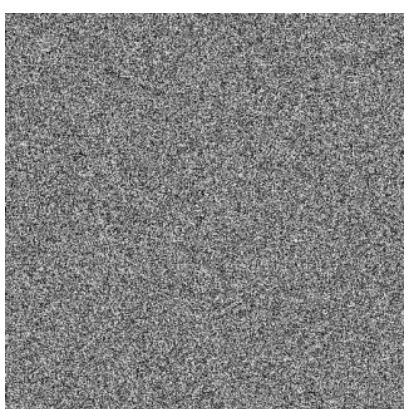

(a)

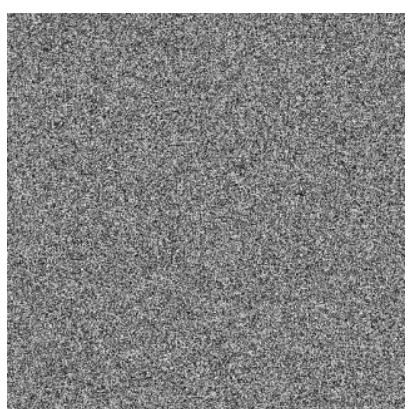

(b)
Fig. 11 Phase profiles, (a) $\phi_{1}$ and (b) $\phi_{2}$ of two wave fields, $U_{1}$ and $U_{2}$ decomposed from the binary image in Fig. 4.

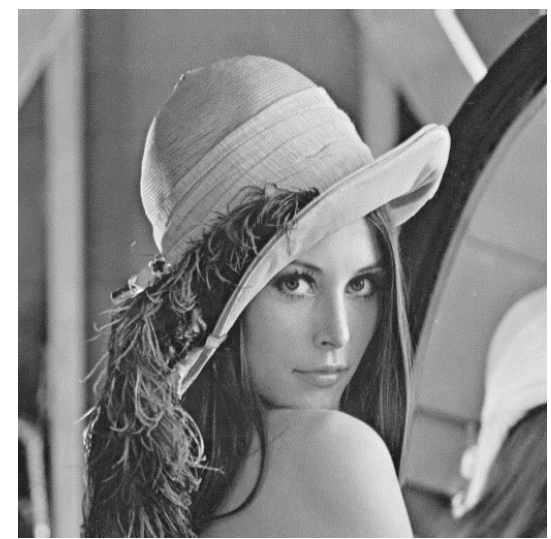

Fig. 12 Decrypted image by interferometric superposition between Fig. 11(a) and (b). 


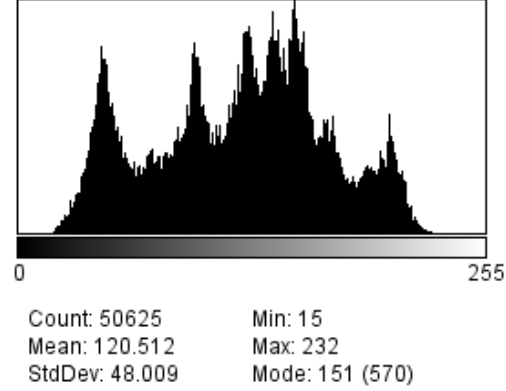

(a)

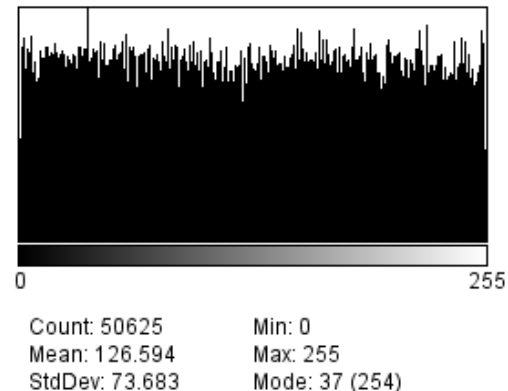

(b)

Fig. 13 The histogram of (a) original grayscale image and (b) decomposed phase profile.

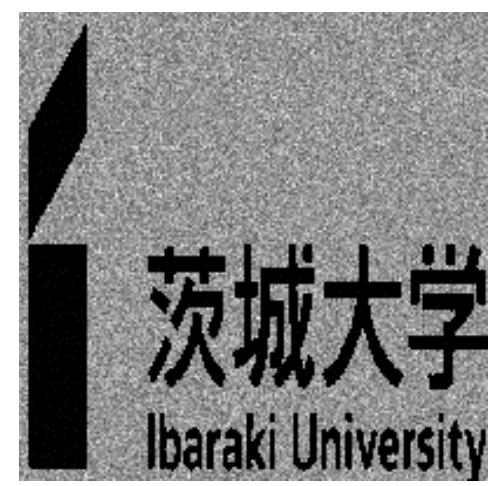

(a)

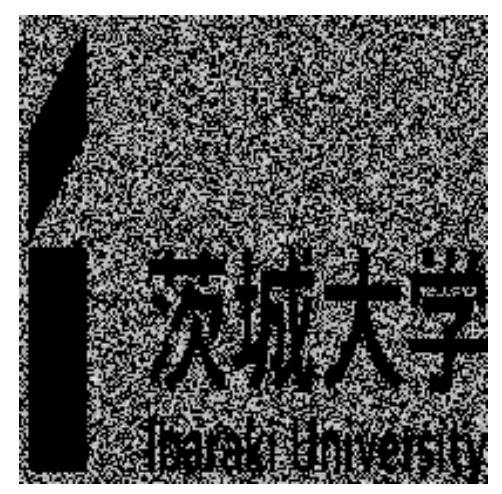

(b)

Fig. 14 Decrypted image decoded from shares with noise, (a) proposed method and (b) random grid method.
Table 1 The mean square error between the original image and the decrypted image. Encrypted image was Fig. 3. The standard deviation of Gaussian noise is represented by $\sigma$.

\begin{tabular}{|c|c|c|}
\hline \multirow{2}{*}{$\sigma$} & \multicolumn{2}{|c|}{ MSE } \\
\cline { 2 - 3 } & Proposed method & Random grid \\
\hline 0 & $4.66^{*} 10^{-30}$ & 0.392 \\
\hline 0.1 & 0.136 & 0.396 \\
\hline
\end{tabular}

\section{Conclusions}

We proposed new encryption technique that utilized wave nature of light for VC. For encryption, the pixel brightness was decomposed to two phasors with constant amplitudes and random phases. Two shares made from that phasors had random phase distributions and constant amplitudes. This means that no one can see what the share itself display.

We also confirmed by the simulation that the interferometric technique was available for decryption. The encrypted image was successfully decrypted from the two shares that are superposed each other as wave fields. It is noteworthy that the decrypted images were almost the same as the original images. This implies that the proposed technique is able to recover the image without noise.

We estimated noise sensitivity of proposed method. If the shares contain some noise, they may bring significantly deteriorated result.

We have succeeded to perform the image encryption and decryption against not only binary images but also grayscale images. This means that the proposed technique is applicable to all types of images. Our method may advance $\mathrm{VC}$ to the industrial level. We are proceeding this study further with some experiments.

\section{References}

(1) M. Naor, and A. Shamir : "Visual cryptography," in Proc. Adv. Cryptol.:EUROCRYPT, vol. 950, pp. 1-12, 1995

(2) O. Kafri, and E. Keren : "Encryption of pictures and shapes by random grids", Optics Letters, vol. 12, No. 6, pp. 377-379, 1987

(3) R-Z. Wang, Y-C. Lana, Y-K Lee, S-Y. Huang, S-J. Shyu, and T-L. Chia : "Incrementing visual cryptography using random grids", Optics Communications, Vol. 283, pp. 4242-4249, 2010 
(4) T-H. Chena, Y-S. Lee, W-L. Huang, J. S-T. Juan, Y-Y. Chen, and M-J Li : "Quality-adaptive visual secret sharing by random grids", The Journal of Systems and Software, Vol. 86, pp. 1267-1274, 2013

(5) X. Wu, and W. Sun : "Improving the visual quality of random grid-based visual secret sharing”, Signal Processing, Vol. 93, pp. 977-995, 2013

(6) X. Wu, and W. Sun : "Random grid-based visual secret sharing with abilities of OR and XOR decryptions", J. Vis. Commun. Image R., Vol. 24, pp. 48-62, 2013

(7) X. Wu, T. Liu, and W. Sun : "Improving the visual quality of random grid-based visual secret sharing via error diffusion", J. Vis. Commun. Image R., Vol. 24, pp. 552-566, 2013

(8) Y-C. Hou, and Z-Y. Quan : "Progressive Visual Cryptography with Unexpanded Shares", IEEE Transactions on Circuits and Systems for Video Technology, Vol. 21, No. 11, pp. 1760-1764, 2011

(9) Y-S. Lee, B-J. Wang, and T-H. Chen : "Quality-improved threshold visual secret sharing scheme by random grids", IET Image Process., Vol. 7, No. 2, pp. 137-143, 2013

(10) S-S. Lee, J-C. Na, S-W. Sohn, C. Park, D-H. Seo, and S-J. Kim : "Visual Cryptography Based on an Interferometric Encryption Technique", ETRI Journal, Vol. 24, No. 5, pp. 373-380, 2002 